\title{
Towards large genus asymtotics of intersection numbers on moduli spaces of curves
}

\author{
Maryam Mirzakhani, Peter Zograf
}

December 7, 2011

\begin{abstract}
We explicitly compute the diverging factor in the large genus asymptotics of the Weil-Petersson volumes of the moduli spaces of $n$-pointed complex algebraic curves. Modulo a universal multiplicative constant we prove the existence of a complete asymptotic expansion of the WeilPetersson volumes in the inverse powers of the genus with coefficients that are polynomials in $n$. This is done by analyzing various recursions for the more general intersection numbers of tautological classes, whose large genus asymptotic behavior is also extensively studied.
\end{abstract}

\section{Introduction and statement of results}

In this note, we study the asymptotic behavior of the Weil-Petersson volumes $V_{g, n}$ of the moduli spaces $\mathcal{M}_{g, n}$ of $n$-pointed complex algebraic curves of genus $g$ as $g \rightarrow \infty$. Here

$$
V_{g, n}=\frac{1}{(3 g-3+n) !} \int_{\mathcal{M}_{g, n}} \omega_{g, n}^{3 g-3+n},
$$

where $\omega_{g, n}$ is the Weil-Petersson symplectic form on $\mathcal{M}_{g, n}$.

The following conjecture was made in $[\mathrm{Z}]$ on the basis of numerical data:

Conjecture 1.1. For any fixed $n \geq 0$

$$
V_{g, n}=(2 g-3+n) !\left(4 \pi^{2}\right)^{2 g-3+n} \frac{1}{\sqrt{g \pi}}\left(1+\frac{c_{n}}{g}+O\left(\frac{1}{g^{2}}\right)\right)
$$

as $g \rightarrow \infty$.

The objective of this paper is to prove the statements formulated below.

Theorem 1.2. There exists a universal constant $C \in(0, \infty)$ such that for any given $k \geq 1, n \geq 0$,

$$
V_{g, n}=C \frac{(2 g-3+n) !\left(4 \pi^{2}\right)^{2 g-3+n}}{\sqrt{g}}\left(1+\frac{c_{n}^{(1)}}{g}+\ldots+\frac{c_{n}^{(k)}}{g^{k}}+O\left(\frac{1}{g^{k+1}}\right)\right),
$$


as $g \rightarrow \infty$. Each term $c_{n}^{(i)}$ in the asymptotic expansion is a polynomial in $n$ of degree $2 i$ with coefficients in $\mathbb{Q}\left[\pi^{-2}, \pi^{2}\right]$ that are effectively computable. Moreover, the leading term of $c_{n}^{(i)}$ is equal to $\frac{(-1)^{i}}{i !\left(2 \pi^{2}\right)^{i}} n^{2 i}$.

Remark 1.3. Note that Conjecture 1.1 claims that $C=\frac{1}{\sqrt{\pi}}$. Numerical data suggest that the coefficients of $c_{n}^{(i)}$ actually belong to $\mathbb{Q}\left[\pi^{-2}\right]$. For example,

$$
c_{n}^{(1)}=-\frac{n^{2}}{2 \pi^{2}}-\left(\frac{1}{4}-\frac{5}{2 \pi^{2}}\right) n+\frac{7}{12}-\frac{17}{6 \pi^{2}} .
$$

Our method also implies that given $k \geq 1, n \geq 0$ we have

$$
\begin{aligned}
& \frac{V_{g, n+1}}{8 \pi^{2} g V_{g, n}}=1+\frac{a_{n}^{(1)}}{g}+\ldots+\frac{a_{n}^{(k)}}{g^{k}}+O\left(\frac{1}{g^{k+1}}\right), \\
& \frac{V_{g-1, n+2}}{V_{g, n}}=1+\frac{b_{n}^{(1)}}{g}+\ldots+\frac{b_{n}^{(k)}}{g^{k}}+O\left(\frac{1}{g^{k+1}}\right),
\end{aligned}
$$

as $g \rightarrow \infty$, where the coefficients $a_{n}^{(i)}$ and $b_{n}^{(i)}$ can be explicitly computed. However, here we do this only for $a_{n}^{(1)}$ and $b_{n}^{(1)}$ :

Theorem 1.4. For any fixed $n \geq 0$ :

$$
\begin{aligned}
& \frac{V_{g, n+1}}{8 \pi^{2} g V_{g, n}}=1+\left(\left(\frac{1}{2}-\frac{1}{\pi^{2}}\right) n-\frac{5}{4}+\frac{2}{\pi^{2}}\right) \cdot \frac{1}{g}+O\left(\frac{1}{g^{2}}\right), \\
& \frac{V_{g-1, n+2}}{V_{g, n}}=1+\frac{3-2 n}{\pi^{2}} \cdot \frac{1}{g}+O\left(\frac{1}{g^{2}}\right) .
\end{aligned}
$$

With the help of the identity $\frac{V_{g+1, n}}{V_{g, n}}=\frac{V_{g+1, n}}{V_{g, n+2}} \cdot \frac{V_{g, n+2}}{V_{g, n+1}} \cdot \frac{V_{g, n+1}}{V_{g, n}}$ this theorem immediately yields

Corollary 1.5. Let $n \geq 0$ be fixed, then

$$
\begin{aligned}
& \quad \frac{V_{g+1, n}}{V_{g, n}}=\left(4 \pi^{2}\right)^{2}(2 g+n-1)(2 g+n-2)\left(1-\frac{1}{2 g}\right) \cdot\left(1+\frac{r_{n}^{(2)}}{g^{2}}+O\left(\frac{1}{g^{3}}\right)\right) \text {, } \\
& \text { as } g \rightarrow \infty \text {. }
\end{aligned}
$$

Note that $r_{n}^{(2)}=3 / 8-c_{n}^{(1)}$ by Theorem [1.2, so that

$$
r_{n}^{(2)}=\frac{n^{2}}{2 \pi^{2}}+\left(\frac{1}{4}-\frac{5}{2 \pi^{2}}\right) n-\frac{5}{24}+\frac{17}{6 \pi^{2}} .
$$

Remark 1.6. Since $\prod_{g=1}^{\infty}\left(1+a_{g}\right)$ converges when $a_{g}=O\left(1 / g^{2}\right)$, Corollary 1.5 easily implies that there exists

$$
\lim _{g \rightarrow \infty} \frac{V_{g, n} \sqrt{g}}{(2 g-3+n) !\left(4 \pi^{2}\right)^{2 g-3+n}}=C \in(0, \infty) .
$$


The proof of Theorem 1.2 is based on (1.1) and (1.2) (see Theorems 4.1, 4.2) and follows similar lines as well. The method we use also allows to calculate the error terms explicitly: even though we only do the calculation for the coefficient of $1 / g$, the error terms of order of $1 / g^{s}$ can be written in terms of values of the intersections of $\psi$ classes on surfaces of genus at most $s$. However, this method does not provide any information about the exact value of $C$.

Analyzing the signs of the error terms of order $1 / g^{2}$ in Theorem 1.4 we get

Corollary 1.7. Given $n \geq 2$, there exists $g_{0}$ such that the sequence

$$
\left\{\frac{V_{g-1, n+2}}{V_{g, n}}\right\}_{g \geq g_{0}}
$$

is increasing. Similarly, for $n \geq 3$ there exists $g_{0}$ such that the sequence

$$
\left\{\frac{8 \pi^{2} g V_{g, n}}{V_{g, n+1}}\right\}_{g \geq g_{0}}
$$

is increasing.

We also obtain somewhat weaker results when $n$ varies as $g \rightarrow \infty$ :

Theorem 1.8. For any sequence $\{n(g)\}_{g=1}^{\infty}$ of non-negative integers with

$$
\lim _{g \rightarrow \infty} \frac{n(g)^{2}}{g}=0
$$

we have

$$
V_{g, n(g)}=\frac{C}{\sqrt{g}}(2 g-3+n(g)) !\left(4 \pi^{2}\right)^{2 g-3+n(g)}\left(1+O\left(\frac{1+n(g)^{2}}{g}\right)\right)
$$

as $g \rightarrow \infty$.

In fact, we prove that

$$
\frac{\sqrt{g} V_{g, n(g)}}{C(2 g-3+n(g)) !\left(4 \pi^{2}\right)^{2 g-3+n(g)}}=O\left(\frac{(g+n) !}{g ! g^{n}}\right) .
$$

\section{Notes and remarks.}

- It may be instructive to compare Theorem 1.2 to the asymptotic formula for the Weil-Petersson volumes for fixed $g \geq 0$ and $n \rightarrow \infty$ (cf. [MZ, Theorem 6.1):

Theorem 1.9. For any fixed $g \geq 0$

$$
V_{g, n}=n ! C^{n} n^{(5 g-7) / 2}\left(c_{g}^{(0)}+\frac{c_{g}^{(1)}}{n}+\ldots\right),
$$

as $n \rightarrow \infty$, where $C=-\frac{2}{x_{0} J_{0}^{\prime}\left(x_{0}\right)}, x_{0}$ is the first positive zero of the Bessel function $J_{0}$, and the coefficients $c_{g}^{(0)}, c_{g}^{(1)}, \ldots$ are effectively computable. 
- Penner $[\mathrm{Pe}$ developed a different method for computing Weil-Petersson volumes by integrating the Weil-Petersson volume form over simplices in the cellular decomposition of the moduli space. In $\mathrm{Gr}$, this method of integration was used to prove that for a fixed $n>0$ there are constants $C_{1}, C_{2}>0$ such that

$$
C_{1}^{g} \cdot(2 g) !<V_{g, n}<C_{2}^{g} \cdot(2 g) !
$$

(This result was extended to the case of $n=0$ by an algebro-geometric argument in $[\mathrm{ST}$.) Note that these estimates do not give much information about the growth of $V_{g-1, n+2} / V_{g, n}$ and $V_{g, n} / V_{g, n+1}$ when $g \rightarrow \infty$.

- The estimates from [M3 imply that given $n \geq 0$ there exists $m>0$ such that

$$
g^{-m} \leq \frac{V_{g, n}}{(2 g-3+n) !\left(4 \pi^{2}\right)^{2 g-3+n)}} \leq g^{m} .
$$

In general,

$$
\lim _{g+n \rightarrow \infty} \frac{\log \left(V_{g, n}\right)}{(2 g+n) \log (2 g+n)}=1
$$

but understanding the asymptotics of $V_{g, n}$ for arbitrary $g, n$ seems to be more complicated.

\section{Relations between intersection numbers}

To begin with, let us recall some well-known facts about tautological classes on $\overline{\mathcal{M}}_{g, n}$ and their intersections. For $n>0$, there are $n$ tautological line bundles $\mathcal{L}_{i}$ on $\overline{\mathcal{M}}_{g, n}$ whose fiber at the point $\left(C, x_{1}, \ldots, x_{n}\right) \in \overline{\mathcal{M}}_{g, n}$ is the cotangent line to $C$ at $x_{i}$, and we put $\psi_{i}=c_{1}\left(\mathcal{L}_{i}\right) \in H_{2}\left(\overline{\mathcal{M}}_{g, n}, \mathbb{Q}\right)$ (cf. e.g. [HM] or [AC]). Notation. For $d=\left(d_{1}, \ldots, d_{n}\right)$ with $d_{i} \in \mathbb{Z}_{\geq 0}$ put $|d|=d_{1}+\ldots+d_{n}$ and, assuming $|d| \leq 3 g-3+n$, put $d_{0}=3 g-3+n-|d|$. Define

$$
\begin{aligned}
{\left[\tau_{d_{1}} \ldots \tau_{d_{n}}\right]_{g, n}=\left[\prod_{i=1}^{n} \tau_{d_{i}}\right]_{g, n} } & =\frac{\prod_{i=1}^{n} 2^{2 d_{i}}\left(2 d_{i}+1\right) ! !}{d_{0} !} \int_{\overline{\mathcal{M}}_{g, n}} \psi_{1}^{d_{1}} \cdots \psi_{n}^{d_{n}} \omega_{g, n}^{d_{0}} \\
& =\prod_{i=1}^{n} 2^{2 d_{i}}\left(2 d_{i}+1\right) ! ! \frac{\left(2 \pi^{2}\right)^{d_{0}}}{d_{0} !} \int_{\overline{\mathcal{M}}_{g, n}} \psi_{1}^{d_{1}} \cdots \psi_{n}^{d_{n}} \kappa_{1}^{d_{0}}
\end{aligned}
$$

where $\kappa_{1}=\frac{\left[\omega_{g, n}\right]}{2 \pi^{2}}$ is the first Mumford class on $\overline{\mathcal{M}}_{g, n}$. According to [M1, for $L=\left(L_{1}, \ldots, L_{n}\right)$ the Weil-Petersson volume of the moduli space of hyperbolic surfaces of genus $g$ with $n>0$ geodesic boundary components of lengths $2 L_{1}, \ldots, 2 L_{n}$ can be written as

$$
V_{g, n}(2 L)=\sum_{\substack{d_{1}, \ldots, d_{n} \\|d| \leq 3 g-3+n}}\left[\prod_{i=1}^{n} \tau_{d_{i}}\right]_{g, n} \frac{L_{1}^{2 d_{1}}}{\left(2 d_{1}+1\right) !} \cdots \frac{L_{n}^{2 d_{n}}}{\left(2 d_{n}+1\right) !} .
$$


Recursive formulas. The following recursions for the intersection numbers $\left[\tau_{d_{1}} \ldots \tau_{d_{n}}\right]_{g, n}$ hold:

(Ia)

$$
\begin{aligned}
& {\left[\tau_{0} \tau_{1} \prod_{i=1}^{n} \tau_{d_{i}}\right]_{g, n+2}=} {\left[\tau_{0}^{4} \prod_{i=1}^{n} \tau_{d_{i}}\right]_{g-1, n+4}+} \\
&+6 \sum_{\substack{g_{1}+g_{2}=g \\
I \amalg J=\{1, \ldots, n\}}}\left[\tau_{0}^{2} \prod_{i \in I} \tau_{d_{i}}\right]_{g_{1},|I|+2} \cdot\left[\tau_{0}^{2} \prod_{i \in J} \tau_{d_{i}}\right]_{g_{2},|J|+2}
\end{aligned}
$$

(Ib)

$$
\begin{array}{r}
{\left[\tau_{0}^{2} \tau_{l+1} \prod_{i=1}^{n} \tau_{d_{i}}\right]_{g, n+3}=\left[\tau_{0}^{4} \tau_{l} \prod_{i=1}^{n} \tau_{d_{i}}\right]_{g-1, n+5}+} \\
+8 \sum_{\substack{g_{1}+g_{2}=g \\
I \amalg J=\{1, \ldots, n\}}}\left[\tau_{0}^{2} \tau_{l} \prod_{i \in I} \tau_{d_{i}}\right]_{g_{1},|I|+3} \cdot\left[\tau_{0}^{2} \prod_{i \in J} \tau_{d_{i}}\right]_{g_{2},|J|+2}+ \\
+4 \sum_{\substack{g_{1}+g_{2}=g \\
I \amalg J=\{1, \ldots, n\}}}\left[\tau_{0} \tau_{l} \prod_{i \in I} \tau_{d_{i}}\right]_{g_{1},|I|+2} \cdot\left[\tau_{0}^{3} \prod_{i \in J} \tau_{d_{i}}\right]_{g_{2},|J|+3} .
\end{array}
$$

(II) $\quad(2 g-2+n)\left[\prod_{i=1}^{n} \tau_{d_{i}}\right]_{g, n}=\frac{1}{2} \sum_{l=1}^{3 g-2+n} \frac{(-1)^{l-1} l \pi^{2 l-2}}{(2 l+1) !}\left[\tau_{l} \prod_{i=1}^{n} \tau_{d_{i}}\right]_{g, n+1}$.

(III) Put $a_{i}=\left(1-2^{1-2 i}\right) \zeta(2 i)$, where $\zeta$ is the Riemann zeta function and $i \in \mathbb{Z}_{\geq 0}$. Then

$$
\left[\tau_{d_{1}} \ldots \tau_{d_{n}}\right]_{g, n}=A_{d}+B_{d}+C_{d},
$$

where

$$
\begin{aligned}
& A_{d}=8 \sum_{j=2}^{n} \sum_{l=0}^{d_{0}}\left(2 d_{j}+1\right) a_{l}\left[\tau_{d_{1}+d_{j}+l-1} \prod_{i \neq 1, j} \tau_{d_{i}}\right]_{g, n-1} \\
& B_{d}=16 \sum_{l=0}^{d_{0}} \sum_{\substack{k_{1}+k_{2}=\\
=l+d_{1}-2}} a_{l}\left[\tau_{k_{1}} \tau_{k_{2}} \prod_{i \neq 1} \tau_{d_{i}}\right]_{g-1, n+1}, \\
& C_{d}=16 \sum_{\substack{g_{1}+g_{2}=g \\
I \amalg J=\{1, \ldots, n\}}} \sum_{\substack{l=0 \\
d_{0}}} \sum_{\substack{k_{1}+k_{2}=\\
=l+d_{1}-2}} a_{l}\left[\tau_{k_{1}} \prod_{i \in I} \tau_{d_{i}}\right]_{g_{1},|I|+1} \cdot\left[\tau_{k_{2}} \prod_{i \in J} \tau_{d_{i}}\right]_{g_{2},|J|+1} .
\end{aligned}
$$

Basic properties of the sequence $\left\{a_{i}=\left(1-2^{1-2 i}\right) \zeta(2 i)\right\}$. It is easy to check that for $i \geq 1$

$$
a_{i}=\frac{1}{(2 i-1) !} \int_{0}^{\infty} \frac{t^{2 i-1}}{1+e^{t}} d t
$$


and

$$
a_{i+1}-a_{i}=\int_{0}^{\infty} \frac{1}{\left(1+e^{t}\right)^{2}}\left(\frac{t^{2 i+1}}{(2 i+1) !}+\frac{t^{2 i}}{2 i !}\right) d t .
$$

Lemma 2.1. The sequence $\left\{a_{i}\right\}_{i=1}^{\infty}$ is increasing. Moreover,

(i)

$$
\lim _{i \rightarrow \infty} a_{i}=1, \quad \sum_{i=0}^{\infty}\left(a_{i+1}-a_{i}\right)=\frac{1}{2}
$$

(ii) $a_{i+1}-a_{i}$ has the order of $1 / 2^{2 i}$, i.e., there exist $C>0$ such that

$$
\frac{1}{C \cdot 2^{2 i}}<a_{i+1}-a_{i}<\frac{C}{2^{2 i}}
$$

(iii)

$$
\sum_{i=0}^{\infty} i\left(a_{i+1}-a_{i}\right)=\frac{1}{4}
$$

(iv) for $j \in \mathbb{Z}, j \geq 2$, the sum

$$
\sum_{i=0}^{\infty} i^{j}\left(a_{i+1}-a_{i}\right)
$$

is a polynomial in $\pi^{2}$ of degree $[j / 2]$ with rational coefficients.

Proof. Both $(i)$ and (ii) easily follow from the definition of $a_{i}$ and (2.5). As for $($ iii), let

$$
S_{1}=\sum_{i=0}^{\infty} \int_{0}^{\infty} \frac{1}{\left(1+e^{t}\right)^{2}} \cdot \frac{t^{2 i+1}}{(2 i+1) !} d t
$$

and

$$
S_{2}=\int_{0}^{\infty} \frac{t e^{t}}{\left(1+e^{t}\right)^{2}} d t
$$

We have

$$
S_{1}=\int_{0}^{\infty} \frac{\left(e^{t}-e^{-t}\right) d t}{2\left(1+e^{t}\right)^{2}}=-\frac{1}{2}+\log 2, \quad S_{2}=\log 2,
$$

so that from (2.5),

$$
2 \sum_{i=1}^{\infty} i\left(a_{i+1}-a_{i}\right)+S_{1}=S_{2}
$$

which implies (2.7). 
We will prove $(i v)$ by induction in $j$. The base case $j=1$ being checked in (iii), we observe that

$$
\begin{gathered}
\sum_{i=0}^{\infty} \frac{i^{j} t^{2 i+1}}{(2 i+1) !}=\left(t D t^{-1}\right)^{j}(\sinh t)=\sum_{l=0}^{j} t^{l}\left(a_{1, j}^{(l)} \cosh t+b_{1, j}^{(l)} \sinh t\right) \\
\sum_{i=0}^{\infty} \frac{i^{j} t^{2 i}}{(2 i) !}=D^{j}(\cosh t)=\sum_{l=0}^{j} t^{l}\left(a_{2, j}^{(l)} \cosh t+b_{2, j}^{(l)} \sinh t\right)
\end{gathered}
$$

where $D=\frac{t}{2} \cdot \frac{d}{d t}$, and the coefficients $a_{*, j}^{(l)}, b_{*, j}^{(l)}$ are rational numbers. A standard computation shows that

$$
\begin{aligned}
& \int_{0}^{\infty} \frac{t^{l} e^{-t}}{\left(1+e^{t}\right)^{2}} d t=l !\left(1-2\left(1-2^{-l}\right) \zeta(l+1)+\left(1-2^{1-l}\right) \zeta(l)\right), \\
& \int_{0}^{\infty} \frac{t^{l} e^{t}}{\left(1+e^{t}\right)^{2}} d t=l !\left(1-2^{1-l}\right) \zeta(l) .
\end{aligned}
$$

From here we see that the values of the zeta function at odd $l=2 k+1$ do not contribute to the sum in $(i v)$ if and only if

$$
(2 k+1) \cdot\left(a_{1, j}^{(2 k+1)}+a_{2, j}^{(2 k+1)}\right)-a_{1, j}^{(2 k)}-a_{2, j}^{(2 k)}+b_{1, j}^{(2 k)}+b_{2, j}^{(2 k)}=0 .
$$

The condition (2.8) is not hard to verify by induction from $j$ to $j+1$. Thus, only the values of the zeta function at even $l$ contribute to the sum.

\section{References.}

- The relationship between the Weil-Petersson volumes and the intersection numbers of $\psi$-classes on $\overline{\mathcal{M}}_{g, n}$ is discussed in [W] and [AC]. An explicit formula for the volumes in terms of the intersections of $\psi$-classes was given in [KMZ, cf. also $\mathrm{MZ}$.

- Recursion (Ia) is a special case of Proposition 3.3 in [LX1]. Similarly, recursion (Ib) is a simple corollary of Propositions 3.3 and 3.4 in [LX1].

- For different proofs of (II) see [DN] and [XX1]. In terms of the volume polynomial $V_{g, n}(L)$, recursion (II) can be written as follows ([DN]):

$$
\frac{\partial V_{g, n+1}}{\partial L_{n+1}}\left(L_{1}, \ldots, L_{n}, 2 \pi \sqrt{-1}\right)=2 \pi \sqrt{-1}(2 g-2+n) V_{g, n}\left(L_{1}, \ldots, L_{n}\right) .
$$

When $n=0$,

$$
V_{g, 1}(2 \pi \sqrt{-1})=0
$$

and

$$
\frac{\partial V_{g, 1}}{\partial L}(2 \pi \sqrt{-1})=2 \pi \sqrt{-1}(2 g-2) V_{g, 0} .
$$


- For a proof of (III) see [M2]; note that (III) applies only when $n>0$ (in case of $n=0$, formula (2.9) gives the necessary estimates on the growth of $\left.V_{g, 0}\right)$. In fact, (III) can be interpreted as a recursive formula for the volumes of moduli spaces $\mathcal{M}_{g, n}(L)$ of hyperbolic surfaces of genus $g$ with $n$ geodesic boundary components of lengths $L_{1}, \ldots, L_{n}$ that describes a removal of a pair of pants on a surface containing at least one of its boundary components. Although (III) is written here in purely combinatorial terms, it is related to the topology of different pant decompositions of a surface, cf. also [Mc] and [LX2].

- When $d_{1}+\ldots+d_{n}=3 g-3+n$, recursion (III) reduces to the Virasoro constraints for the intersection numbers of $\psi_{i}$-classes predicted by Witten W], cf. also [MS. For different proofs and discussions of these relations see, [Ko, $\mathrm{OP}$, [M1], $\mathrm{KL}$, and [EO].

- In this paper, we are mainly interested in the intersection numbers of $\kappa_{1}$ and $\psi_{i}$ classes. For generalizations of (III) to the case of intersection numbers involving higher Mumford's $\kappa$-classes see [LX1], [E] and [Ka].

\section{Asymptotics of intersection numbers when $n$ is fixed}

In this section, we prove Theorem 1.4 This theorem implies that there exists $C \in(0, \infty)$ such that

$$
\lim _{g \rightarrow \infty} \frac{V_{g, n} \sqrt{g}}{(2 g-3+n) !\left(4 \pi^{2}\right)^{2 g-3+n}}=C .
$$

This result will be generalized in 4 .

We recall that for any $n \geq 0$ the results obtained in $\mathrm{M} 3$ yield

$$
\frac{V_{g, n}}{8 \pi^{2} g V_{g-1, n+1}}=1+O\left(\frac{1}{g}\right)
$$

and

$$
\frac{V_{g, n}}{V_{g-1, n+2}}=1+O\left(\frac{1}{g}\right)
$$

as $g \rightarrow \infty$. The main ingredient of the proof is the following property of the intersection numbers:

$$
\left[\tau_{d_{1}} \ldots \tau_{d_{n}}\right]_{g, n} \leq\left[\tau_{0}^{n}\right]_{g, n}=V_{g, n}
$$

for any $d=\left(d_{1}, \ldots, d_{n}\right)$. Moreover,

$$
\frac{\left[\tau_{d_{1}} \ldots \tau_{d_{n}}\right]_{g, n}}{V_{g, n}}=1+O\left(\frac{1}{g}\right),
$$

as $g \rightarrow \infty$. 
Remark 3.1. The same result holds if $d_{1}, \ldots, d_{n}$ grow slowly with $g$ in such a way that

$$
\frac{d_{1} \ldots d_{n}}{g} \rightarrow 0
$$

as $g \rightarrow \infty$. In particular, (3.5) holds if $d_{i}=O(\log g)$ for each $i=1, \ldots, n$.

A stronger statement is formulated below:

Theorem 3.2. Let $k, n \geq 1$, then

(i)

$$
\frac{\left[\tau_{k} \tau_{0}^{n-1}\right]_{g, n}}{V_{g, n}}=1+\frac{e_{n, k}^{(1)}}{g}+O\left(\frac{1}{g^{2}}\right),
$$

as $g \rightarrow \infty$, where

$$
e_{n, k}^{(1)}=-\frac{k^{2}+(n-5 / 2) k-n / 2+3 / 2}{\pi^{2}} .
$$

(ii)

$$
\begin{aligned}
8 n\left[\tau_{k-1} \tau_{0}^{n-2}\right]_{g, n-1} & <\left[\tau_{k} \tau_{0}^{n-1}\right]_{g, n}-\left[\tau_{k+1} \tau_{0}^{n-1}\right]_{g, n} \leq \\
& \leq 16\left((n+k) V_{g, n-1}+k V_{g-1, n+1}\right)
\end{aligned}
$$

Remark 3.3. In general, one can show that for $k \geq 1$

$$
\frac{\left[\tau_{k+1} \tau_{j_{1}} \ldots \tau_{j_{s}} \tau_{0}^{n-1-s}\right]_{g, n}}{\left[\tau_{k} \tau_{j_{1}} \ldots \tau_{j_{s}}, \tau_{0}^{n-1-s}\right]_{g, n}}=1-\frac{2\left(k+j_{1}+\ldots j_{s}\right)+n-3 / 2}{\pi^{2} g}+O\left(\frac{1}{g^{2}}\right) .
$$

Proof of Theorem 3.2. We will need the following simple fact. Let $\left\{r_{i}\right\}_{i=1}^{\infty}$ be a sequence of real numbers and $\left\{k_{g}\right\}_{g=1}^{\infty}$ be an increasing sequence of positive integers. Assume that for all $g$, and $i$ we have $0 \leq c_{i, g} \leq c_{i}$, and $\lim _{g \rightarrow \infty} c_{i, g}=c_{i}$. If $\sum_{i=1}^{\infty}\left|c_{i} r_{i}\right|<\infty$, then

$$
\lim _{g \rightarrow \infty} \sum_{i=1}^{k_{g}} r_{i} c_{i, g}=\sum_{i=1}^{\infty} r_{i} c_{i} .
$$

To prove part $(i)$ of the Theorem, it is sufficient to show that

$$
\frac{\left[\tau_{1} \tau_{0}^{n-1}\right]_{g, n}}{\left[\tau_{0}^{n}\right]_{g, n}}=1-\frac{n}{2 \pi^{2} g}+O\left(\frac{1}{g^{2}}\right)
$$

and for $k \geq 1$

$$
\frac{\left[\tau_{k+1} \tau_{0}^{n-1}\right]_{g, n}}{\left[\tau_{k} \tau_{0}^{n-1}\right]_{g, n}}=1-\frac{2 k+n-3 / 2}{\pi^{2} g}+O\left(\frac{1}{g^{2}}\right)
$$


Here we use the recursive formula (III) to expand the difference $\left[\tau_{k} \tau_{0}^{n-1}\right]_{g, n}-$ $\left[\tau_{k+1} \tau_{0}^{n-1}\right]_{g, n}$ in terms of the intersection numbers on $\overline{\mathcal{M}}_{g-1, n+1}, \overline{\mathcal{M}}_{g, n-1}$ and $\overline{\mathcal{M}}_{g_{1}, n_{1}} \times \overline{\mathcal{M}}_{g_{2}, n_{2}}$. For the sake of brevity let us put

$$
\left[\tau_{k} \tau_{0}^{n-1}\right]_{g, n}-\left[\tau_{k+1} \tau_{0}^{n-1}\right]_{g, n}=\widetilde{A}_{k, g, n}+\widetilde{B}_{k, g, n}+\widetilde{C}_{k, g, n},
$$

where $\widetilde{A}_{k, g, n}, \widetilde{B}_{k, g, n}$, and $\widetilde{C}_{k, g, n}$ are the terms corresponding to (2.2), (2.3) and (2.4) respectively. We will evaluate these terms separately.

1. Contribution from (2.2). By (III), the numbers

$$
\left[\tau_{k-1} \tau_{0}^{n-2}\right]_{g, n-1}, \ldots,\left[\tau_{3 g+n-4} \tau_{0}^{n-2}\right]_{g, n-1}
$$

contribute to $\left[\tau_{k} \tau_{0}^{n-1}\right]_{g, n}$ and $\left[\tau_{k+1} \tau_{0}^{n-1}\right]_{g, n}$. In fact, it is easy to check that

$$
\widetilde{A}_{k, g, n}=8 a_{0}\left[\tau_{k-1} \tau_{0}^{n-2}\right]_{g, n-1}+8 \sum_{i=1}^{3 g-3+n-k}\left(a_{i+1}-a_{i}\right)\left[\tau_{k-1+i} \tau_{0}^{n-2}\right]_{g, n-1} .
$$

The term $\left[\tau_{k-1} \tau_{0}^{n-2}\right]_{g, n-1}$ is non-zero only when $k \geq 1$. In order to calculate the asymptotic behavior of $\widetilde{A}_{k, g, n} / V_{g, n-1}$ we simply apply (3.5) and (3.6). In view of Lemma 2.1, we get that

$$
\lim _{g \rightarrow \infty} \frac{\widetilde{A}_{k, g, n}}{V_{g, n-1}}=8(n-1)\left(a_{0} \delta+\sum_{i=0}^{\infty}\left(a_{i+1}-a_{i}\right)\right)=8(n-1)(1 / 2 \delta+1 / 2),
$$

where $\delta=0$ when $k=0$, and otherwise $\delta=1$. Thus

$$
\frac{\widetilde{A}_{k, g, n}}{V_{g, n-1}}=8(n-1)(1 / 2 \delta+1 / 2)+O\left(\frac{1}{g}\right)
$$

as $g \rightarrow \infty$. On the other hand, Lemma 2.1 also implies that

$$
\widetilde{A}_{k, g, n} \leq 8(n-1) V_{g, n-1} .
$$

2. Contribution from (2.3). Similarly, by (III), the numbers $\left[\tau_{i} \tau_{j} \tau_{0}^{n-1}\right]_{g-1, n+1}$ contribute to $\left[\tau_{k} \tau_{0}^{n-1}\right]_{g, n}$ (resp. to $\left[\tau_{k+1} \tau_{0}^{n-1}\right]_{g, n}$ ) whenever $i+j \geq k-2$ (resp. $i+j \geq k-1)$. To simplify the notation, let

$$
T_{m, g, n}=\sum_{i+j=m}\left[\tau_{i} \tau_{j} \tau_{0}^{n-1}\right]_{g-1, n+1}
$$

(we assume $T_{m, g, n}=0$ for $m<0$ ). Then

$\widetilde{B}_{k, g, n}=16\left(a_{0} T_{k-2, g, n}+\left(a_{1}-a_{0}\right) T_{k-1, g, n}+\ldots+\left(a_{i+1}-a_{i}\right) T_{k-1+i, g, n}+\ldots\right)$.

Note that by (3.5) as $g \rightarrow \infty$,

$$
\lim _{g \rightarrow \infty} \frac{T_{m, g, n}}{V_{g-1, n+1}}=m+1 .
$$


Now since $a_{0}=1 / 2$, Lemma 2.1 (iii), together with (3.5) and (3.6), implies for $k>1$

$$
\begin{aligned}
\lim _{g \rightarrow \infty} \frac{\widetilde{B}_{k, g, n}}{V_{g-1, n+1}} & =16\left(a_{0}(k-1)+\sum_{i=0}^{\infty}(i+k)\left(a_{i+1}-a_{i}\right)\right)= \\
& =16\left(\frac{k-1}{2}+\frac{k}{2}+1 / 4\right)=16(k-1+1 / 4), \\
\lim _{g \rightarrow \infty} \frac{\widetilde{B}_{1, g, n}}{V_{g-1, n+1}} & =16 \sum_{i=0}^{\infty}(i+1)\left(a_{i+1}-a_{i}\right)=4,
\end{aligned}
$$

and we also have

$$
\widetilde{B}_{k, g, n} \leq 16 k V_{g-1, n+1} .
$$

3. Contribution from (2.4). By the results obtained in [M3]

$$
\sum_{\substack{I \amalg J=\{2, \ldots, n\} \\ 0 \leq g^{\prime} \leq g}} \frac{V_{g^{\prime},|I|+1} \cdot V_{g-g^{\prime},|J|+1}}{V_{g, n}}=O\left(\frac{1}{g^{2}}\right) .
$$

Put

$$
S_{k_{1}, k_{2}, g, n}=\sum_{\substack{I \amalg J=\{2, \ldots, n\} \\ 0 \leq g^{\prime} \leq g}}\left[\tau_{k_{1}} \prod_{i \in I} \tau_{i}\right]_{g^{\prime},|I|+1} \cdot\left[\tau_{k_{2}} \prod_{i \in J} \tau_{i}\right]_{g-g^{\prime},|J|+1} .
$$

Note that by (3.4) and recursion (Ia),

$$
S_{k_{1}, k_{2}, g, n} \leq \sum_{\substack{I \amalg J=\{2, \ldots, n\} \\ 0 \leq g^{\prime} \leq g}} V_{g^{\prime},|I|+1} \cdot V_{g-g^{\prime},|J|+1} \leq V_{g, n-1}
$$

Therefore, the contribution from the term (2.4) in (III) satisfies

$$
\begin{aligned}
0 & \leq \widetilde{C}_{k, g, n} \leq 16 \sum_{i=0}^{\infty}\left(a_{i}-a_{i+1}\right) \sum_{k_{1}+k_{2}=i+k} S_{k_{1}, k_{2}, g, n} \leq \\
& \leq 16 \sum_{i=0}^{\infty}(i+k)\left(a_{i}-a_{i+1}\right) V_{g, n-1} \leq 16(1 / 4+k / 2) V_{g, n-1} .
\end{aligned}
$$

Using (3.6), as in the cases $\mathbf{1}$ and $\mathbf{2}$ considered above, we see that the contribution from the term (2.4) in (III) becomes small as $g \rightarrow \infty$ :

$$
\frac{\widetilde{C}_{k, g, n}}{V_{g, n}}=O\left(\frac{1}{g^{2}}\right)
$$

and

$$
\widetilde{C}_{k, g, n} \leq 16(1+k) V_{g, n-1}
$$


Now, in view of (3.2), (3.3) and (3.5), equations (3.9), (3.11) and (3.13)) imply that for $k \geq 1$

$$
1-\frac{\left[\tau_{k+1} \tau_{0}^{n-1}\right]_{g, n}}{\left[\tau_{k} \tau_{0}^{n-1}\right]_{g, n}}=\frac{2 k+n-3 / 2}{\pi^{2}} \cdot \frac{1}{g}+O\left(\frac{1}{g^{2}}\right)
$$

and

$$
1-\frac{\left[\tau_{1} \tau_{0}^{n-1}\right]_{g, n}}{\left[\tau_{0}^{n}\right]_{g, n}}=\frac{n}{2 \pi^{2}} \cdot \frac{1}{g}+O\left(\frac{1}{g^{2}}\right) .
$$

Finally, the inequalities (3.10), (3.12) and (3.14) imply part (ii) of the Theorem.

Proof of Theorem 1.4. We start with proving (1.3). From (II),

$$
\frac{2(2 g-2+n) V_{g, n}}{V_{g, n+1}}=\sum_{l=1}^{3 g-2+n} \frac{(-1)^{l-1} l \pi^{2 l-2}}{(2 l+1) !} \cdot \frac{\left[\tau_{l}, \tau_{0}^{n}\right]_{g, n+1}}{V_{g, n+1}} .
$$

Differentiating $t^{-1} \sin t$ and putting $t=\pi$ we get

$$
\sum_{l=1}^{\infty} \frac{(-1)^{l-1} l \pi^{2 l-2}}{(2 l+1) !}=\frac{1}{2 \pi^{2}}
$$

Now we can use (3.6), and Theorem 3.2 in order to calculate the error term in $(2 g-2+n) V_{g, n} / V_{g, n+1}-1 / 4 \pi^{2}$. Clearly,

$$
\frac{(2 g-2+n) V_{g, n}}{V_{g, n+1}}-\frac{1}{4 \pi^{2}}=\sum_{l=1}^{3 g-2+n} \frac{(-1)^{l-1} l \pi^{2 l-2}}{(2 l+1) !} \cdot\left(\frac{\left[\tau_{l} \tau_{0}^{n}\right]_{g, n+1}}{V_{g, n+1}}-1\right) .
$$

Then Theorem 3.2, (i) implies that

$$
\begin{aligned}
\frac{(2 g-2+n) V_{g, n}}{V_{g, n+1}} & -\frac{1}{4 \pi^{2}}= \\
= & -\sum_{l=1}^{\infty} \frac{(-1)^{l-1}\left(l^{2}+(n-3 / 2) l-n+1\right) l \pi^{2 l-2}}{2(2 l+1) ! \pi^{2}} \cdot \frac{1}{g}+O\left(\frac{1}{g^{2}}\right) .
\end{aligned}
$$

On the other hand,

$$
\sum_{l=1}^{\infty} \frac{(-1)^{l-1} l\left(l^{2}+(n-3 / 2) l-n / 2+1\right) \pi^{2 l}}{(2 l+1) !}=-\frac{4 n+\pi^{2}-8}{8 \pi^{2}} .
$$

Hence,

$$
\frac{4 \pi^{2}(2 g-2+n) V_{g, n}}{V_{g, n+1}}=1+\frac{4 n+\pi^{2}-8}{4 \pi^{2}} \cdot \frac{1}{g}+O\left(\frac{1}{g^{2}}\right),
$$

and

$$
\frac{8 \pi^{2} g V_{g, n}}{V_{g, n+1}}=1+\left(\left(\frac{1}{\pi^{2}}-\frac{1}{2}\right) n+\frac{5}{4}-\frac{2}{\pi^{2}}\right) \cdot \frac{1}{g}+O\left(\frac{1}{g^{2}}\right) .
$$


We proceed with proving (1.4). First, we will check this estimate when $n \geq 2$, that is,

$$
\frac{V_{g-1, n+4}}{V_{g, n+2}}=1-\frac{2 n+1}{\pi^{2}} \cdot \frac{1}{g}+O\left(\frac{1}{g^{2}}\right) .
$$

From the recursion (Ia) we get

$$
\begin{aligned}
\frac{V_{g-1, n+4}}{V_{g, n+2}}=\frac{\left[\tau_{1} \tau_{0}^{n+1}\right]_{g, n+2}}{V_{g, n+2}}- \\
-\frac{6}{V_{g, n+2}} \sum_{\substack{g_{1}+g_{2}=g \\
I \amalg J=\{1, \ldots, n\}}} V_{g_{1},|I|+2} \cdot V_{g_{2},|J|+2} .
\end{aligned}
$$

On the other hand, by Theorem 3.2 we have for $k=1$

$$
\frac{\left[\tau_{1} \tau_{0}^{n+1}\right]_{g, n+2}}{\left[\tau_{0}^{n+2}\right]_{g, n+2}}=1-\frac{n+2}{2 \pi^{2}} \cdot \frac{1}{g}+O\left(\frac{1}{g^{2}}\right)
$$

and (3.3) implies

$$
\begin{aligned}
\frac{1}{V_{g, n+2}} \sum_{\substack{g_{1}+g_{2}=g \\
I \amalg J=\{1, \ldots, n\}}} V_{g_{1},|I|+2} \cdot V_{g_{2},|J|+2} & =2 n \frac{V_{g, n+1}}{V_{g, n+2}}+O\left(\frac{1}{g^{2}}\right) \\
& =\frac{2 n}{8 \pi^{2}} \cdot \frac{1}{g}+O\left(\frac{1}{g^{2}}\right) .
\end{aligned}
$$

Hence

$$
\frac{V_{g-1, n+4}}{V_{g, n+2}}=1-\left(\frac{n+2}{2 \pi^{2}}+\frac{12 n}{8 \pi^{2}}\right) \cdot \frac{1}{g}+O\left(\frac{1}{g^{2}}\right)=1-\frac{2 n+1}{\pi^{2} g}+O\left(\frac{1}{g^{2}}\right) .
$$

The remaining cases $n=0,1$ follow from (1.3) and (1.4) for $n \geq 2$. For instance, if $n=1$

$$
\frac{V_{g-1,3}}{V_{g, 1}}=\frac{V_{g-1,4}}{V_{g, 2}} \cdot \frac{V_{g, 2}}{V_{g, 1}} \cdot \frac{V_{g-1,3}}{V_{g-1,4}}=1+\frac{1}{\pi^{2}} \cdot \frac{1}{g}+O\left(\frac{1}{g^{2}}\right) .
$$

The case $n=0$ can be treated similarly.

Theorem 1.4 immediately implies Corollary 1.5 about the asymptotic behavior of the ratio $V_{g+1, n} / V_{g, n}$ (see Introduction). An important consequence of Corollary 1.5. explained in Remark 1.6. is formula (3.1) announced at the beginning of this Section. As a byproduct of this statement we also get the following estimate that we will need later:

Lemma 3.4. Fix $n_{1}, n_{2}, s \geq 0$. Then

$$
\sum_{\substack{g_{1}+g_{2}=g \\ 2 g_{i}+n_{i} \geq s, i=1,2}} V_{g_{1}, n_{1}} \cdot V_{g_{2}, n_{2}}=O\left(\frac{V_{g, n_{1}+n_{2}}}{g^{s}}\right) .
$$




\section{Error terms in the asymptotics expansions}

In this section, we prove Theorem 1.2 using the following results:

Theorem 4.1. We have the following asymptotic expansions as $g \rightarrow \infty$ :

(i) Given the integers $n, s \geq 1$, and $d=\left(d_{1}, \ldots, d_{n}\right)$, there exist $e_{n, d}^{(1)}, \ldots e_{n, d}^{(s-1)}$ independent of $g$ such that

$$
\frac{\left[\tau_{d_{1}} \ldots \tau_{d_{n}}\right]_{g, n}}{V_{g, n}}=1+\frac{e_{n, d}^{(1)}}{g}+\ldots+\frac{e_{n, d}^{(s-1)}}{g^{s-1}}+O\left(\frac{1}{g^{s}}\right)
$$

(ii) Given $n \geq 0, s \geq 1$, there exist $a_{n}^{(i)}, b_{n}^{(i)} i=1, \ldots, s-1$, independent of $g$ such that

$$
\begin{aligned}
\frac{4 \pi^{2}(2 g-2+n) V_{g, n}}{V_{g, n+1}} & =1+\frac{a_{n}^{(1)}}{g}+\ldots+\frac{a_{n}^{(s-1)}}{g^{s-1}}+O\left(\frac{1}{g^{s}}\right) \\
\frac{V_{g, n}}{V_{g-1, n+2}} & =1+\frac{b_{n}^{(1)}}{g}+\ldots+\frac{b_{n}^{(s-1)}}{g^{s-1}}+O\left(\frac{1}{g^{s}}\right) .
\end{aligned}
$$

The coefficients of the above asymptotic expansions (4.1)-4.3) can be characterized more precisely:

Theorem 4.2. We have

(i) For any fixed $n$ and $d$ the coefficient $e_{n, d}^{(i)}$ is a polynomial in $\mathbb{Q}\left[\pi^{-2}, \pi^{2}\right]$ of degree at most $i$.

(ii) Each $a_{n}^{(i)}$ and $b_{n}^{(i)}$ is a polynomial in $\mathbb{Q}\left[n, \pi^{-2}, \pi^{2}\right]$ of degree $i$ in $n$ and of degree at most $i$ in $\pi^{-2}$ and $\pi^{2}$.

Remark 4.3. In the simplest case $\left[\tau_{0} \tau_{k}\right]_{g, 2} / V_{g, 2}$ we have the following expansions:

$$
\begin{aligned}
\frac{\left[\tau_{0} \tau_{1}\right]_{g, 2}}{V_{g, 2}} & =1-\frac{1}{\pi^{2} g}+\left(\frac{1}{64}-\frac{5}{6 \pi^{2}}+\frac{1}{\pi^{4}}\right) \cdot \frac{1}{g^{2}}+O\left(\frac{1}{g^{3}}\right) \\
\frac{\left[\tau_{0} \tau_{2}\right]_{g, 2}}{V_{g, 2}} & =1-\frac{7}{2 \pi^{2} g}+\left(\frac{1}{64}-\frac{13}{6 \pi^{2}}+\frac{1}{\pi^{4}}\right) \cdot \frac{1}{g^{2}}+O\left(\frac{1}{g^{3}}\right), \\
\frac{\left[\tau_{0} \tau_{k}\right]_{g, 2}}{V_{g, 2}} & =1-\frac{2 k^{2}-k+1}{2 \pi^{2} g}+\left(\frac{k^{4}}{2 \pi^{4}}-\frac{13 k^{3}}{6 \pi^{4}}-\left(\frac{1}{2 \pi^{2}}-\frac{27}{8 \pi^{4}}\right) \cdot k^{2}\right. \\
& \left.+\left(\frac{1}{24 \pi^{2}}-\frac{59}{24 \pi^{4}}\right) \cdot k+\left(\frac{1}{64}-\frac{1}{4 \pi^{2}}+\frac{19}{8 \pi^{4}}\right)\right) \cdot \frac{1}{g^{2}}+O\left(\frac{1}{g^{3}}\right) .
\end{aligned}
$$

We see that no (positive) powers of $\pi^{2}$ appear in these expansions. The term of order $1 / g^{2}$ is a polynomial in $k$ of degree 4 for $k \geq 3$ (computed numerically). However, for $k=1,2$ the general formula is off by $\frac{1}{8 \pi^{2}}+\frac{5}{8 \pi^{4}}$ and $\frac{5}{8 \pi^{4}}$ for $k=1$ and $k=2$ respectively. This is a manifestation of the "boundary effect" in recursion (III). These results can be proved using Remark 3.3. 
Remark 4.4. Note that a result similar to (4.2) and (4.3) holds for the inverse ratios $V_{g, n+1} /\left(8 \pi^{2} g V_{g, n}\right)$ and $V_{g-1, n+2} / V_{g, n}$. This is because of the following simple fact. Let $\left\{w_{g}\right\}_{g=1}^{\infty}$ be a sequence of the form

$$
w_{g}=1+\frac{u_{1}}{g}+\ldots+\frac{u_{s-1}}{g^{s-1}}+O\left(\frac{1}{g^{s}}\right),
$$

then

$$
\frac{1}{w_{g}}=1+\frac{v_{1}}{g}+\ldots+\frac{v_{s-1}}{g^{s-1}}+O\left(\frac{1}{g^{s}}\right)
$$

where each $v_{i}$ is a polynomial in $u_{1}, \ldots, u_{i}$ with integer coefficients. Moreover, if $u_{i}$ is a polynomial of degree $m_{i}$ in $n$, then $v_{k}$ is a polynomial of degree at

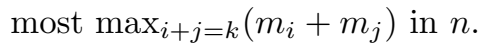

Let us first outline some general ideas underlying the proofs of Theorems 4.1 and 4.2. All proofs are by induction in $s$ and are similar to each other. We basically follow the same steps as in the course of proving Theorems 3.2 and 1.4 .

Remark 4.5. Let $f: \mathbb{Z}_{+} \rightarrow \mathbb{R}$ be a function such that $\lim _{g \rightarrow \infty} f(g)$ exists. We say that $f$ has an expansion up to $O\left(1 / g^{s}\right)$ if there exist $e_{0}, \ldots, e_{s-1} \in \mathbb{R}$ so that

$$
f(g)=e_{0}+\frac{e_{1}}{g}+\ldots+\frac{e_{s-1}}{g^{s-1}}+O\left(\frac{1}{g^{s}}\right) .
$$

Note that if $f_{1}, \ldots, f_{k}$ all have expansions of order $s$, the expansion of the product $f_{1} \cdots f_{k}$ up to $1 / g^{s}$ can easily be calculated in terms of the expansions of $f_{i}$.

In this section, we are interested in the expansions of the ratios $\frac{V_{g, n}}{V_{g-1, n+2}}$, $\frac{4 \pi^{2}(2 g-2+n) V_{g, n}}{V_{g, n+1}}$ and $\frac{\left[\tau_{d_{1}} \ldots \tau_{d_{n}}\right]_{g, n}}{V_{g, n}}$. Some remarks are in order:

1. In general, given $g^{\prime}, n^{\prime}$, in order to obtain the expansion of $\frac{V_{g-g^{\prime}, n-n^{\prime}}}{V_{g, n}}$ up to $O\left(1 / g^{s}\right)$, it is enough to know the expansions of $\frac{V_{g, k}}{V_{g-1, k+2}}$ and $\frac{4 \pi^{2}(2 g-2+k) V_{g, k}}{V_{g, k+1}}$ up to $O\left(1 / g^{s-2 g^{\prime}-n^{\prime}}\right)$; this is simply because

$$
\begin{aligned}
& \frac{V_{g-g^{\prime}, n-n^{\prime}}}{V_{g, n}}=\prod_{j=-n^{\prime}+1}^{2 g^{\prime}} \frac{4 \pi^{2}\left(2 g-2 g^{\prime}+n-j+1\right) V_{g-g^{\prime}, n+j-1}}{V_{g-g^{\prime}, n+j}} . \\
& \cdot \prod_{j=1}^{g^{\prime}} \frac{V_{g-j, n+2 j}}{V_{g-j+1, n+2 j-2}} \cdot \prod_{j=-n^{\prime}+1}^{2 g^{\prime}} \frac{1}{\left(4 \pi^{2}\left(2 g-2 g^{\prime}+n-j+1\right)\right)} .
\end{aligned}
$$

2. Following (Ia) and (II), the expansion of $\frac{4 \pi^{2}(2 g-2+n) V_{g, n}}{V_{g, n+1}}$ up to $O\left(1 / g^{s}\right)$ can be written explicitly in terms of the expansion of $\frac{\left[\tau_{l} \tau_{0}^{n}\right]_{g, n+1}}{V_{g, n+1}}$ up to $O\left(1 / g^{s}\right)$; see 
(3.15). Similarly, by (3.8) and (4.4) the expansion of $\frac{V_{g, n}}{V_{g-1, n+2}}$ up to $O\left(1 / g^{s}\right)$ can be written in terms of the expansions of $\frac{V_{g, n_{1}}}{V_{g-1, n_{1}+2}}$ and $\frac{4 \pi^{2}\left(2 g-2+n_{1}\right) V_{g, n_{1}}}{V_{g, n_{1}+1}}$ up to $O\left(1 / g^{s-1}\right)$, and the expansion of $\frac{\left[\tau_{1} \tau_{0}^{n-1}\right]_{g, n}}{V_{g, n}}$ up to $O\left(1 / g^{s}\right)$.

3. In view of (III), the expansion of $\frac{\left[\tau_{d_{1}} \ldots \tau_{d_{n}}\right]_{g, n}}{V_{g, n}}$ up to $O\left(1 / g^{s}\right)$ can be written in terms of the expansions of $\frac{\left[\tau_{c_{1} \ldots \tau_{c m}}\right]_{g, m}}{V_{g, m}}$ up to $O\left(1 / g^{s-1}\right)$ and the expansions of $\frac{V_{g, n}}{V_{g-1, n+2}}$ and $\frac{4 \pi^{2}(2 g-2+n) V_{g, n}}{V_{g, n+1}}$ up to $O\left(1 / g^{s-1}\right)$. Actually, for our purposes it will be enough to obtain the expansion of $\frac{\left[\tau_{d_{1}+1} \ldots \tau_{d_{n}}\right]_{g, n}}{\left[\tau_{d_{1}} \ldots \tau_{d_{n}}\right] g, n}$ up to $O\left(1 / g^{s}\right)$. As in (3.8), we put

$$
\left[\tau_{d_{1}} \ldots \tau_{d_{n}}\right]_{g, n}-\left[\tau_{d_{1}+1} \ldots \tau_{d_{n}}\right]_{g, n}=\widetilde{A}_{d, g, n}+\widetilde{B}_{d, g, n}+\widetilde{C}_{d, g, n},
$$

where $\widetilde{A}_{d, g, n}, \widetilde{B}_{d, g, n}$, and $\widetilde{C}_{d, g, n}$ are the terms corresponding to (2.2), (2.3) and (2.4). Put

$$
\frac{\left[\tau_{d_{1}} \ldots \tau_{d_{n}}\right]_{g, n}-\left[\tau_{d_{1}+1} \ldots \tau_{d_{n}}\right]_{g, n}}{V_{g, n}}=S_{1}+S_{2}+S_{3}
$$

where

$$
\begin{aligned}
& S_{1}=\frac{1}{4 \pi^{2}(2 g-3+n)} \cdot \frac{4 \pi^{2}(2 g-3+n) V_{g, n-1}}{V_{g, n}} \cdot \frac{\widetilde{A}_{d, g, n}}{V_{g, n-1}}, \\
& S_{2}=\frac{1}{4 \pi^{2}(2 g-3+n)} \cdot \frac{4 \pi^{2}(2 g-3+n) V_{g-1, n+1}}{V_{g-1, n+2}} \cdot \frac{V_{g-1, n+2}}{V_{g, n}} \cdot \frac{\widetilde{B}_{d, g, n}}{V_{g-1, n+1}}, \\
& S_{3}=\frac{\widetilde{C}_{d, g, n}}{V_{g, n}} .
\end{aligned}
$$

Similar to the case $\mathbf{1}$ in the proof of Theorem 3.2 we have

$$
\frac{\widetilde{A}_{d, g, n}}{V_{g, n-1}}=8 \sum_{i=0}^{3 g-3+n-d_{1}}\left(a_{i+1}-a_{i}\right) \frac{\left[\tau_{d_{1}+d_{j}+i-1} \tau_{d_{2}} \ldots \widehat{\tau_{d_{j}}} \ldots \tau_{d_{n}}\right]_{g, n-1}}{V_{g, n-1}}
$$

(the hat means that the corresponding entry is omitted, and $a_{-1}=0$ ). The case $\mathbf{2}$ of the same proof now reads

$$
\frac{\widetilde{B}_{d, g, n}}{V_{g-1, n+1}}=16\left(a_{0} T_{k-2, g, n}+\left(a_{1}-a_{0}\right) T_{k-1, g, n}+\ldots+\left(a_{i+1}-a_{i}\right) T_{k-1+i, g, n}+\ldots\right),
$$

where

$$
T_{m, g, n}=\frac{\sum_{i+j=m}\left[\tau_{i} \tau_{j} \tau_{d_{2}} \ldots \tau_{d_{n}}\right]_{g-1, n+1}}{V_{g-1, n+1}} .
$$

Similarly, according to (2.4), each term in $\widetilde{C}_{d, g, n}$ has the form

$$
\sum_{\substack{k_{1}+k_{2}=\\=i+d_{1}-2}}\left(a_{i+1}-a_{i}\right)\left[\tau_{k_{1}} \prod_{i \in I} \tau_{d_{i}}\right]_{g^{\prime},|I|+1} \cdot\left[\tau_{k_{2}} \prod_{i \in J} \tau_{d_{i}}\right]_{g-g^{\prime},|J|+1},
$$


where $I \amalg J=\{2, \ldots, n\}$, and $0 \leq g^{\prime} \leq g$. In order to obtain the expansions of $S_{1}$ and $S_{2}$, we can use the expansions of ratios $\frac{\left[\tau_{c_{1}} \ldots \tau_{c_{n-1}}\right]_{g, n-1}}{V_{g, n-1}}$ and $\frac{\left[\tau_{c_{1}} \ldots \tau_{c_{n+1}}\right]_{g-1, n+1}}{V_{g-1, n+1}}$ up to $O\left(1 / g^{s-1}\right)$. What concerns the term $S_{3}$, by Lemma 3.4 each product

$$
\left[\tau_{k_{1}} \prod_{i \in I} \tau_{d_{i}}\right]_{g^{\prime},|I|+1} \cdot\left[\tau_{k_{2}} \prod_{i \in J} \tau_{d_{i}}\right]_{g-g^{\prime},|J|+1}
$$

is of order $O\left(1 / g^{s+1}\right)$ unless either $2 g^{\prime}-1+|I|<s$ or $2 g-2 g^{\prime}+|J|-$ $1<s$. In these cases we apply (4.4) to obtain the expansion of $S_{3}$ up to $O\left(1 / g^{s}\right)$. Then we can use the expansions of $\frac{4 \pi^{2}(2 g-2+n) V_{g-1, n+2}}{V_{g-1, n+3}}$ (for $S_{1}$ ) and $\frac{V_{g, n}}{V_{g-1, n+2}}, \frac{4 \pi^{2}(2 g-3+n) V_{g-1, n+1}}{V_{g-1, n+2}}$ (for $S_{2}$ ), all up to $O\left(1 / g^{s-1}\right)$, to get the expansion of $\frac{\left[\tau_{d_{1}} \ldots \tau_{d_{n}}\right]_{g, n}-\left[\tau_{d_{1}+1} \ldots \tau_{d_{n}}\right]_{g, n}}{V_{g, n}}$ up to $O\left(1 / g^{s}\right)$, which will complete the inductive step.

Remark 4.6. We will need the following basic facts to prove Theorems 4.1 and 4.2

1. For any $k \geq 0$ the sum

$$
\sum_{l=0}^{\infty} \frac{(-1)^{l} l^{k} \pi^{2 l}}{(2 l+1) !}
$$

is a polynomial in $\pi^{2}$ of degree at most $2[k / 2]$ with rational coefficients (this can be easily seen by expanding $\frac{\sin x}{x}$ in the Taylor series, differentiating it and putting $x=\pi$ ).

2. For a polynomial $p(x)=\sum_{j=1}^{m} b_{j} x^{j}$ of degree $m$,

$$
\tilde{p}(x)=\sum_{i=1}^{\infty}\left(a_{i+1}-a_{i}\right) p(x+i)
$$

is again a polynomial of degree $m$. The coefficient of $\tilde{p}(x)$ at $x^{j}$ is equal to $\sum_{j+r \leq m}\left(\begin{array}{c}j+r \\ j\end{array}\right) b_{j+r} \cdot A(r)$ where $A(r)=\sum_{i=1}^{\infty} i^{r}\left(a_{i+1}-a_{i}\right)$.

3. Since $\psi_{i}$ and $\kappa_{1}=\frac{\left[\omega_{g, n}\right]}{2 \pi^{2}}$ are rational classes (i.e., belong to $H^{2}\left(\overline{\mathcal{M}}_{g, n}, \mathbb{Q}\right)$, cf. $[\mathrm{Wo},[\mathrm{AC}]$ ),

$$
\left[\tau_{d_{1}} \ldots \tau_{d_{n}}\right]_{g, n} \in \mathbb{Q} \cdot \pi^{6 g-6+2 n-2|d|},
$$

where $|d|=\sum_{i=1}^{n} d_{i}$. In particular, $V_{g, n}=\left[\tau_{0} \ldots \tau_{0}\right]_{g, n}$ is a rational multiple of $\pi^{6 g-6+2 n}$ ([Wo, see also [M2] for a different point of view).

4. The function $S_{m}(n)=\sum_{i=1}^{n} i^{m}$ is a polynomial in $n$ of degree $m+1$ with rational coefficients (Faulhaber's formula). 
Proof of Theorem 4.1 First, we use (Ia), (II) and (III) to prove the existence of $e_{n, d}^{(s)}, a_{n}^{(s)}$ and $b_{n}^{(s)}$. This is similar to what we did in the proofs of (1.3) and (1.4). In fact, instead of $(i)$ we will prove a stronger statement: namely, there exist polynomials $Q_{n}^{(s)}\left(d_{1}, \ldots, d_{n}\right)$ and $q_{n}^{(s)}\left(d_{1}, \ldots, d_{n}\right)$ in variables $d_{1}, \ldots, d_{n}$ of degrees $s+1$ and $s$ respectively such that for any $d=\left(d_{1}, \ldots, d_{n}\right)$

$$
\left|\frac{\left[\tau_{d_{1}} \ldots \tau_{d_{n}}\right]_{g, n}}{V_{g, n}}-1-\frac{e_{n, d}^{(1)}}{g}-\ldots-\frac{e_{n, d}^{(s)}}{g^{s}}\right| \leq \frac{Q_{n}^{(s)}\left(d_{1}, \ldots, d_{n}\right)}{g^{s+1}},
$$

and

$$
\left|e_{n, d}^{(s)}\right| \leq q_{n}^{s}\left(d_{1}, \ldots, d_{n}\right) .
$$

These formulas follow from the two claims below:

Claim 1: Formulas (4.9) and (4.10) for $s=r$ and formulas (4.2), (4.3) for $s<r$ imply (4.2) and (4.3) for $s=r$.

In fact, from (3.15), (4.9) and (4.10) for $d=(l, 0, \ldots, 0)$ we have

$$
\frac{(2 g-2+n) V_{g, n}}{V_{g, n+1}}-\frac{1}{4 \pi^{2}}=\frac{a_{n}^{(1)}}{g}+\ldots+\frac{a_{n}^{(s)}}{g^{s}}+O\left(\frac{1}{g^{s+1}}\right),
$$

where

$$
a_{n}^{(s)}=\sum_{l=1}^{\infty} \frac{(-1)^{l-1} l \pi^{2 l-2}}{(2 l+1) !} e_{n, l}^{(s)}
$$

with $d=(l, 0, \ldots, 0)$. The existence of $a_{n}^{(s)}$ is guaranteed by the estimate

$$
\sum_{l=N}^{\infty} \frac{(-1)^{l-1} l^{k+1} \pi^{2 l-2}}{(2 l+1) !}=O\left(e^{-N}\right)
$$

valid for any $k \geq 0$.

Similarly, we can use (3.17) to evaluate the error term in the expansion of $\frac{V_{g-1, n+4}}{V_{g, n+2}}-1$. In this case we apply (4.9) with $d=(1,0, \ldots, 0)$. Note that by Lemma 3.4

$$
\begin{aligned}
& \frac{6}{V_{g, n+2}} \sum_{\substack{g_{1}+g_{2}=g \\
I \amalg J=\{1, \ldots, n\}}} V_{g_{1},|I|+2} \cdot V_{g_{2},|J|+2}= \\
& \quad=\sum_{2 j+i+2 \leq s}\left(\begin{array}{c}
n \\
i
\end{array}\right) \frac{V_{g-j, n+2-i}}{V_{g, n+2}} \times V_{j, i+2}+O\left(\frac{1}{g^{s+1}}\right) .
\end{aligned}
$$

We can now use (4.4), and together with (4.2) and (4.3) for $s=r$ this yields the expansion of $\frac{V_{g-j, n+2-i}}{V_{g, n+2}}$ up to $O\left(1 / g^{r+1}\right)$.

Claim 2. Fromulas (4.2), (4.3), (4.9) and (4.10) for $s<r$ imply (4.9) and (4.10) for $s=r$. 
According to (4.5), we need to evaluate the contributions from the term $S_{1}$, $S_{2}$ and $S_{3}$ up to $O\left(1 / g^{r}\right)$. In view of (4.6), (4.7) and (4.8) we can use (4.9) for $s=r-1$ to obtain the expansions of $\frac{\widetilde{A}_{d, g, n}}{V_{g, n-1}}$ and $\frac{\widetilde{B}_{d, g, n}}{V_{g-1, n+1}}$ up to $O\left(1 / g^{r-1}\right)$. Formula (4.6) now takes the form

$$
\frac{\widetilde{A}_{d, g, n}}{V_{g, n-1}}=8 \sum_{i=0}^{\infty}\left(a_{i+1}-a_{i}\right)\left(1+\frac{e_{n, d(i)}^{(1)}}{g}+\ldots+\frac{e_{n, d^{\prime}}^{(r-1)}}{g^{r-1}}+E_{d^{\prime}, r}\right),
$$

where $d(i)=d_{1}+d_{j}+i-1, d_{2}, \ldots \widehat{d_{j}} \ldots d_{n}$, and $E_{d^{\prime}, r} \leq \frac{Q_{n}^{r-1}\left(d^{\prime}\right)}{g^{r}}$. Note that by Lemma 2.1, (ii)

$$
\sum_{i=N}^{\infty}\left(a_{i+1}-a_{i}\right) i^{k}=O\left(2^{-N}\right)
$$

This allows us to calculate the contribution from $S_{1}$ up to $O\left(1 / g^{r}\right)$. The other two terms $S_{2}$ and $S_{3}$ can be treated in a similar way.

In order to prove Theorem 4.2 we need two auxiliary lemmas:

Lemma 4.7. (i) Fix $k(0<k \leq n)$ and $d_{1}, \ldots, d_{k} \in \mathbb{Z}_{\geq 0}$. Then for $d=$ $\left(d_{1}, \ldots, d_{k}, 0, \ldots, 0\right)$ each term $e_{n, d}^{(s)}$ in the asymptotic expansion 4.1) is a polynomial in $n$ of degree at most $s$.

(ii) Each $a_{n}^{(s)}$ and $b_{n}^{(s)}$ in (4.2) and 4.3) is a polynomial in $n$ of degree $s$.

Proof. The proof is again by induction on $s$. We prove a slightly stronger version of $(i)$ :

$\left(i^{\prime}\right)$ For given $k$ and $s$, there exist polynomials $q_{j}\left(d_{1}, \ldots, d_{k}\right), j=0, \ldots, s$, such that the term $e_{n, d}^{(s)}$ has the form

$$
e_{n, d}^{(s)}=\sum_{j=0}^{s} e_{d, j} n^{j}
$$

with $\left|e_{d, j}\right| \leq q_{j}\left(d_{1}, \ldots, d_{k}\right)$. In other words, the coefficients of $e_{n, d}^{(s)}$ considered as a polynomial in $n$ grow at most polynomially in $d_{1}, \ldots, d_{k}$. This would imply that

$$
\sum_{i=1}^{\infty}\left(a_{i+1}-a_{i}\right) e_{d(i), j}<\infty
$$

where $d(i)=\left(d_{1}+i, d_{2}, \ldots, d_{k}, 0, \ldots, 0\right)$.

Now, by (3.17) and (3.15) the statement $\left(i^{\prime}\right)$ for $s=r$ implies part (ii) of the Lemma for $s=r$, that is clear in view of (4.12) and (4.13). Moreover, part (ii) for $s=r$ and the statement $\left(i^{\prime}\right)$ for $s<r$ imply $\left(i^{\prime}\right)$ for $s=r$. This follows from (4.5) by analyzing the contributions from the terms $S_{1}, S_{2}$ and $S_{3}$ as in (4.6), (4.7) and (4.8). 
Lemma 4.8. (i) Let $n$ and $k$ be fixed, and let $d=\left(d_{1}, \ldots, d_{k}, d_{k+1}, \ldots, d_{n}\right)$ with $d_{k+1}, \ldots, d_{n}$ fixed. Then there exists a polynomial $P_{s} \in \mathbb{R}\left[x_{1}, \ldots, x_{k}\right]$ (depending on $d_{k+1}, \ldots, d_{n}$ ) of degree $2 s$ such that $e_{n, d}^{(s)}=P_{s}\left(d_{1}, \ldots, d_{k}\right)$ provided $d_{j} \geq 2 s$ for $j=1, \ldots, k$. The coefficient at each monomial $d_{1}^{\alpha_{1}} \cdots d_{k}^{\alpha_{k}}$ in $P_{s}$ is a linear rational combination of $\pi^{2 s-2\left[\frac{|\alpha|+1}{2}\right]}, \ldots, \pi^{-2 s}$, where $|\alpha|=\alpha_{1}+\ldots+\alpha_{k}$. Moreover, for arbitrary $d_{j}$ the difference $e_{n, d}^{(s)}-$ $P_{s}\left(d_{1}, \ldots, d_{k}\right)$ is a linear rational combinations of $\pi^{2 s-2|d|}, \ldots, \pi^{-2 s}$.

(ii) Each $a_{n}^{(s)}$ and $b_{n}^{(s)}$ in (4.2) and (4.3) is a rational polynomial of degree at most s in $\mathbb{Q}\left[\pi^{2}, \pi^{-2}\right]$.

Proof. The proof is by induction in $s$ and utilizes the same techniques that we have already used before, so we only sketch it here. The statement of the lemma follows from the following claims:

Claim 1: Part (ii) for $s<r$ implies that the coefficient at $1 / g^{r}$ in the expansion of $V_{g_{1}, n_{1}+1} \cdot V_{g-g_{1}, n-n_{1}+1} / V_{g, n}$ is a polynomial of degree at most $r$ in $\mathbb{Q}\left[\pi^{2}, \pi^{-2}\right]$. More precisely, when $g_{1}, n_{1}$ and $n_{2}$ are fixed

$$
\frac{V_{g_{1}, n_{1}+1} \cdot V_{g-g_{1}, n-n_{1}+1}}{V_{g, n}}=\sum_{k=2 g_{1}+n_{1}-1}^{s} \frac{c_{g_{1}, n_{1}}^{(k)}}{g^{k}}+O\left(\frac{1}{g^{s+1}}\right),
$$

where $c_{g_{1}, n_{1}}^{(k)}$ is a polynomial of degree at most $s$ in $\mathbb{Q}\left[\pi^{2}, \pi^{-2}\right]$.

This is a simple consequence of Remarks 4.6(2), 4.5(2) and formula (4.4).

Claim 2: Part (i) of the lemma for $s=r$ and part (ii) for $s<r$ imply part (ii) for $s=r$.

Note that by part (i), each element of the infinite sum (4.12) is a polynomial of degree at most $r$ in $\mathbb{Q}\left[\pi^{2}, \pi^{-2}\right]$. On the other hand, when $l \geq r$ the coefficient $e_{n, l}^{(r)}=P_{r}(l)$ is a polynomial in $l$. Remark 4.6(1) and the properties of the coefficients of $P(l)$ imply that $a_{n}^{(r)}$ is a rational polynomial of degree at most $r$ in $\mathbb{Q}\left[\pi^{2}, \pi^{-2}\right]$. The similar statement for $b_{n}^{(r)}$ follows from (3.17), Claim 1 and part (i) for $s=r$ (when $k=1$ and $d=(1,0, \ldots, 0)$ ).

Claim 3: Part (ii) for $s<r$ and part (i) for $s<r$ imply part (i) for $s=r$.

First, we use (Ib) to find the polynomial $P_{r}$. We put $P_{r}\left(d_{1}, \ldots, d_{k}\right)=e_{n+2, d}^{(r)}$ for $d=\left(0,0, d_{1}, \ldots, d_{k}, d_{k+1}, \ldots, d_{n}\right)$ with $d_{1}, \ldots, d_{k} \geq 2 r$. Note that the number $\left[\tau_{l}, \tau_{d_{1}} \ldots \tau_{d_{k}}\right]_{g, k+1} \neq 0$ only when $l \leq 3 g-2+k-|d|,|d|=d_{1}+\ldots+d_{k}$. On the other hand, by Lemma 3.4, the term

$$
\left[\tau_{0}^{2} \tau_{l} \prod_{i \in I} \tau_{d_{i}}\right]_{g_{1},|I|+3} \cdot\left[\tau_{0}^{2} \prod_{i \in J} \tau_{d_{i}}\right]_{g_{2},|J|+2}
$$


in $(\mathbf{I b})$ is of order $O\left(1 / g^{s+1}\right)$ unless either $2 g_{1}+|I|+1<s$ or $2 g_{2}+|J|<s$. Similarly the term

$$
\left[\tau_{0} \tau_{l} \prod_{i \in I} \tau_{d_{i}}\right]_{g_{1},|I|+2} \cdot\left[\tau_{0}^{3} \prod_{i \in J} \tau_{d_{i}}\right]_{g_{2},|J|+3}
$$

in (Ib) is of order $O\left(1 / g^{s+1}\right)$ unless $2 g_{1}+|I|<s$ or $2 g_{2}+|J|+1<s$. For both terms we can explicitly calculate the expansions of the factors up to $O\left(1 / g^{s+1}\right)$ if we know their expansions up to $O\left(1 / g^{s}\right)$.

Then Lemma 3.4, formula (Ib) and the induction hypothesis imply that

$$
P_{s}\left(d_{1}+1, d_{2}, \ldots, d_{k}\right)=P_{s}\left(d_{1}, \ldots, d_{k}, 0,0\right)+P_{s}^{\prime}\left(d_{1}, \ldots, d_{k}\right)+P_{s}^{\prime \prime}\left(d_{1}, \ldots, d_{k}\right),
$$

where $P_{s}^{\prime}$ is a polynomial when $d_{1} \geq s-1, d_{2} \geq s, \ldots, d_{k} \geq s$, and $P_{s}^{\prime \prime}\left(d_{1}, \ldots, d_{k}\right)$ is nontrivial only if $d_{1}, \ldots, d_{k} \leq s$. The result follows from Lemma 4.7 $(i)$ and Claim 1. In the simplest case $k=1, d_{2}=\ldots=d_{n}=0$, and $d_{1}=d \geq 2 s$, the relation $(\mathbf{I} \mathbf{b})$ implies that

$$
\left[\tau_{d} \tau_{0}^{n-1}\right]_{g, n}=\left[\tau_{s} \tau_{0}^{n+2(d-s)-1}\right]_{g-(d-s), n+2(d-s)}+Q(d),
$$

where $Q$ is a polynomial in $d$.

Next, we use (III) to prove the statement about the coefficients of $P_{s}$. We explicitly calculate the expansion of $\left[\tau_{d_{1}} \ldots \tau_{d_{n}}\right]_{g, n}$ using (4.5) and Remark 4.5 We evaluate the contributions from the terms $S_{1}, S_{2}$ and $S_{3}$ and show that there exist polynomials $Q_{i}^{(s)}, i=1,2,3$, such that:

1. When $d_{1}, \ldots, d_{k}$ are large enough (i.e., $\geq 2 s$ ), the coefficient $S_{i}^{(s)}$ at $1 / g^{s}$ in $S_{i}$ is equal to $Q_{i}^{(s)}\left(d_{1}, \ldots, d_{k}\right)$;

2. The coefficient at the monomial $d_{1}^{\alpha_{1}} \cdots d_{k}^{\alpha_{k}}$ in each $Q_{i}^{(s)}$ is a linear rational combination of $\pi^{2 s-2[(|\alpha|+1) / 2]}, \ldots, \pi^{-2 s}$, where $|\alpha|=\alpha_{1}+\ldots+\alpha_{k}$;

3. For all $d_{1}, \ldots, d_{k}$, the difference $S_{i}^{(s)}-Q_{i}^{(s)}\left(d_{1}, \ldots, d_{k}\right)$ is a linear rational combinations of $\left\{\pi^{2 s-2|d|}, \ldots, \pi^{-2 s}\right\}$.

Contributions from $S_{1}$. We use the induction hypothesis and Lemma 2.1 to expand the ratio $\widetilde{A}_{d, g, n} / V_{g, n-1}$ up to the order $O\left(1 / g^{s}\right)$ by expanding each $\left[\tau_{d_{1}+d_{j}+i-1} \tau_{d_{2}} \ldots \widehat{\tau_{d_{j}}} \ldots \tau_{d_{n}}\right]_{g, n-1} / V_{g, n-1}$ up to $O\left(1 / g^{s-1}\right)$. Put

$$
q^{(m)}(d)=8 \sum_{j=2}^{n} \sum_{i=0}^{\infty}\left(a_{i+1}-a_{i}\right) e_{n, d_{j}(i)}^{(m-1)},
$$

where $d_{j}(i)=\left(d_{1}+d_{j}+i-1, d_{2}, \ldots, \widehat{d_{j}} \ldots, d_{n}\right)$. Then following Remark 4.6(2), and (4.14)

$$
S_{1}=\sum_{j=1}^{s} \frac{Q_{1}^{(j)}(d)}{g^{j}}+O\left(\frac{1}{g^{s}}\right),
$$


where

$$
Q_{1}^{(j)}(d)=\sum_{j_{1}+j_{2}=j} q^{\left(j_{1}\right)}(d) \cdot a_{n-1}^{\left(j_{2}\right)} .
$$

Now by the induction hypothesis and part (ii) of the lemma for $s<r$, both $q^{(m)}(d)$ and $Q_{1}^{(m)}(d)$ are polynomials in $d_{1}, \ldots, d_{k}$ of degree $2 m$ whenever $d_{1}, \ldots, d_{k}$ are large enough. The coefficient at $d_{1}^{\alpha_{1}} \ldots d_{k}^{\alpha_{k}}$ in $q^{(m)}(d)$ is a rational linear combination of the terms of the form $c_{\alpha_{j}(r)} \sum_{i=0}^{\infty} i^{r}\left(a_{i+1}-a_{i}\right)$, where $c_{\alpha_{j}(r)}$ is the coefficient at $x_{1}^{r+\alpha_{1}+\alpha_{j}} x_{2}^{\alpha_{2}} \ldots \widehat{x_{j}^{\alpha_{j}}} \ldots x_{k}^{\alpha_{k}}$ in $P^{(m-1)}\left(x_{1}, \ldots, \widehat{x_{j}}, \ldots, x_{k}\right)$. Now by Lemma 2.1 $(i v)$ the sum $\sum_{i=0}^{\infty} i^{r}\left(a_{i+1}-a_{i}\right)$ is a rational linear combination of $\pi^{2}, \ldots, \pi^{2[r / 2]}$, and by the induction hypothesis $c_{\alpha_{j}(r)}$ is a rational linear combination of $\pi^{2 m-2-2 r-|\alpha|} \ldots, \pi^{-2 m-2}$. Therefore the coefficients of these polynomials are rational combinations of $\pi^{2 m-|\alpha|}, \ldots, \pi^{-2 m}$.

Contributions from $S_{2}$. In the same way, the induction hypothesis and Lemma 2.1 allow to expand $\widetilde{B}_{d, g, n} / V_{g-1, n+1}$ up to the order of $1 / g^{s}$. Repeating the proof of case $(\mathbf{2})$ of Theorem 3.2 we see that each term in the expansion is of the form (4.7). In the expansion of $T_{m, g, n}$ the term of order $1 / g^{m}$ is a polynomial in $d_{1}, \ldots, d_{n}$ of degree $m+1$, whose coefficients satisfy the properties 1,2 , and 3 mentioned above.

Contributions from $S_{3}$. What is different here compared to step 3 in the proof of Theorem 3.2. is that $\widetilde{C}_{d, g, n}$ contributes to the terms of order $1 / g^{2}, \ldots, 1 / g^{r}$. However, by Lemma 3.4 these contributions can be evaluated using (4.4). More precisely, as in Remark 4.5, we can write $S_{3}$ as a sum of finitely many elements of the form

$$
\begin{aligned}
\frac{\left[\tau_{k} \prod_{i \in I} \tau_{d_{i}}\right]_{g^{\prime},|I|+1}}{V_{g^{\prime},|I|+1}} & \cdot \frac{V_{g-g^{\prime},|J|+1} \cdot V_{g^{\prime},|I|+1}}{V_{g, n}} \times \\
& \times \sum_{i=0}^{\infty}\left(a_{i+1}-a_{i}\right) \frac{\left[\tau_{d_{1}+i-2-k} \prod_{i \in J} \tau_{d_{i}}\right]_{g-g^{\prime},|J|+1}}{V_{g-g^{\prime},|J|+1}},
\end{aligned}
$$

where $I \amalg J=\{2, \ldots, n\}, 0 \leq g^{\prime} \leq g$, and $2 g^{\prime}-1+|I| \leq r$. The result now follows from the induction hypothesis on the behavior of the coefficient at $1 / g^{s}$ in the expansion of $\frac{\left[\tau_{d_{1}+i-2-k} \prod_{i \in J} \tau_{d_{i}}\right]_{g-g^{\prime},|J|+1}}{V_{g-g^{\prime},|J|+1}}$ for $s<r$, together Claim 1 and Remark 4.6(2), (3).

Proof of Theorem 4.2, It is easy to see that part $(i)$ of the theorem is a special case of Lemma 4.8 for $n=k$. Part (ii) is a consequence of Lemma $4.8(i i)$ and 4.7( $($ ii).

Proof of Theorem 1.2, For a fixed $n \geq 0$, Theorem 4.1 (ii) applied to the 
obvious identity $\frac{V_{g+1, n}}{V_{g, n}}=\frac{V_{g+1, n}}{V_{g, n+2}} \cdot \frac{V_{g, n+2}}{V_{g, n+1}} \cdot \frac{V_{g, n+1}}{V_{g, n}}$ immediately yields

$$
\begin{aligned}
\frac{V_{g+1, n}}{V_{g, n}} & =\left(4 \pi^{2}\right)^{2}(2 g+n-1)(2 g+n-2) \times \\
& \times\left(1-\frac{1}{2 g}\right) \cdot\left(1+\frac{r_{n}^{(2)}}{g^{2}}+\ldots+\frac{r_{n}^{(s)}}{g^{s}}+O\left(\frac{1}{g^{s+1}}\right)\right)
\end{aligned}
$$

as $g \rightarrow \infty$. On the other hand, we have

$$
V_{g, 1}=\prod_{j=2}^{g-1} \frac{V_{j+1,1}}{V_{j, 1}} \cdot V_{2,1},
$$

and therefore the result of Theorem 1.2 for $n=1$ is a consequence of the following:

Lemma 4.9. Let $a_{2}, \ldots, a_{l} \in \mathbb{R}$ and let $\left\{c_{j}\right\}_{j=1}^{\infty}$ be a positive sequence with the property

$$
c_{j}=1+\frac{a_{2}}{j^{2}}+\ldots+\frac{a_{s}}{j^{s}}+O\left(\frac{1}{j^{s+1}}\right) .
$$

Then there exist $b_{1}, \ldots, b_{s-1}$ such that

$$
\prod_{j=1}^{g} c_{j}=C_{0}\left(1+\frac{b_{1}}{g}+\ldots \frac{b_{s-1}}{g^{s-1}}+O\left(\frac{1}{g^{s}}\right)\right),
$$

as $g \rightarrow \infty$, where $C_{0}=\prod_{j=1}^{\infty} c_{j}$. Moreover, $b_{1}, \ldots, b_{s-1}$ are polynomials in $a_{2}, \ldots, a_{s}$ with rational coefficients.

Proof. First, we can write

$$
c_{j}=R_{j}\left(1+\frac{d_{2}}{j^{2}}\right) \ldots\left(1+\frac{d_{l}}{j^{l}}\right),
$$

where $d_{2}, \ldots d_{l}$ are polynomials in $a_{2}, \ldots, a_{l}$ and $R_{j}=1+O\left(1 / j^{l+1}\right)$ as $j \rightarrow \infty$. One can check that there exists a constant $R$ such that

$$
\prod_{j=1}^{g} R_{j}=R\left(1+O\left(\frac{1}{g^{l}}\right)\right)
$$

as $g \rightarrow \infty$. So it is enough to prove that

$$
\prod_{j=1}^{g}\left(1+\frac{d_{k}}{j^{k}}\right)=C_{k}\left(1+\frac{p_{k}^{(1)}}{g}+\ldots \frac{p_{k}^{(l-1)}}{g^{l-1}}+O\left(\frac{1}{g^{l}}\right)\right)
$$

as $g \rightarrow \infty$, where $p_{k}^{(1)}, \ldots, p_{k}^{(l)}$ are polynomials in $d_{k}$ with rational coefficients, and $C_{k}=\prod_{j=1}^{\infty}\left(1+\frac{d_{k}}{j^{k}}\right), k \geq 2$. It is enough to get bounds for the error term

$$
E_{N, k}=\log \prod_{j=N}^{\infty}\left(1+\frac{d_{k}}{j^{k}}\right)=\sum_{j=N}^{\infty} \log \left(1+\frac{d_{k}}{j^{k}}\right) .
$$


Using the Taylor series $\log (1+x)=x-x^{2} / 2+x^{3} / 3-\ldots$, we expand each term $\log \left(1+\frac{d_{k}}{j^{k}}\right)$ up to the order $j^{-l}$.

Now we make use of the Euler-Maclaurin summation formula (cf. [Ed]):

$$
\begin{aligned}
\zeta(s) & =\sum_{i=1}^{N} \frac{1}{i^{s}}+\frac{1}{(s-1) N^{s-1}}+\frac{1}{2 N^{s}}+ \\
& +\sum_{m=1}^{r-1} \frac{s(s+1) \ldots(s+2 m-2) B_{2 m}}{(2 m) ! N^{s+2 m-1}}+E_{2 r}(s),
\end{aligned}
$$

where $\zeta(s)$ is the Riemann zeta function, $B_{2 m}=(-1)^{m+1} 2(2 m) ! \frac{\zeta(2 m)}{(2 \pi)^{2 m}}$ is the $m$ th Bernoulli number, and the error term $E_{2 r}(s)$ has an estimate

$$
\left|E_{2 r}(s)\right|<\left|\frac{s(s+1) \ldots(s+2 r-2) B_{2 r}}{(\operatorname{Re}(s)+2 r-1)(2 r) ! N^{s+2 r-1}}\right|
$$

(the formula holds for all $s$ with $\operatorname{Re}(s)>-2 r+1$ ). From here we get that for any integer $k>1$

$$
\begin{aligned}
\sum_{i=N+1}^{\infty} \frac{1}{i^{k}} & =\frac{1}{(k-1) N^{k-1}}+\frac{1}{2 N^{k}}+ \\
& +\sum_{m=1}^{[(l-k) / 2]} \frac{B_{2 m} \cdot k(k+1) \ldots(k+2 m-2)}{(2 m) ! N^{k+2 m-1}}+O\left(\frac{1}{N^{l}}\right)
\end{aligned}
$$

as $N \rightarrow \infty$. Therefore, given $l$, there exist $q_{k}^{(k-1)}, \ldots, q_{k}^{(l)}$ such that

$$
E_{N, k}=\frac{q_{k}^{(k-1)}}{N^{k-1}}+\ldots+\frac{q_{k}^{(l)}}{N^{l}}+O\left(\frac{1}{N^{l+1}}\right)
$$

where each $q_{k}^{(i)}$ is a polynomial with rational coefficients in $d_{k}$. Now we can easily get hold on the error terms $e^{E_{N, k}}$ :

$$
e^{E_{N, k}}=\frac{p_{k}^{(k-1)}}{N^{k-1}}+\ldots+\frac{p_{k}^{(l)}}{N^{l}}+O\left(\frac{1}{N^{l+1}}\right)
$$

where $p_{k}^{(j)}$ is a polynomial in $q_{k}^{(i)}, i=k-1, \ldots, l$, which implies the result. As a result, we can write

$$
V_{g, 1}=C \frac{(2 g-2) !\left(4 \pi^{2}\right)^{2 g-2}}{\sqrt{g}}\left(1+\frac{c_{1}^{(1)}}{g}+\ldots+\frac{c_{1}^{(k)}}{g^{k}}+O\left(\frac{1}{g^{k+1}}\right)\right),
$$

as $g \rightarrow \infty$. On the other hand,

$$
\frac{V_{g, n}}{C \cdot C_{g, n}}=\prod_{j=1}^{n-1} \frac{V_{g, j+1}}{4 \pi^{2}(2 g-2+j) V_{g, j}} \cdot \frac{V_{g, 1}}{C \cdot C_{g, n}},
$$


where $C_{g, n}=(2 g-3+n) !\left(4 \pi^{2}\right)^{2 g-3+n} g^{-1 / 2}, C=\lim _{g \rightarrow \infty} \frac{V_{g, 1}}{C_{g, 1}}$. The following is elementary:

Fact. Let $\left\{f_{i}\right\}_{i=1}^{\infty}$ be a sequence of functions with the expansion

$$
f_{i}(g)=1+\frac{p(1, i)}{g}+\ldots+\frac{p(s, i)}{g^{s}}+O\left(\frac{1}{g^{s+1}}\right) .
$$

Assume that for a given $j, p(j, k)$ is a polynomial in $k$ of degree $j$. Let

$$
H(g, n)=\prod_{j=1}^{n} f_{j}(g)
$$

Then

$$
H(g, n)=1+\frac{h_{1}(i)}{g}+\ldots+\frac{h_{s}(i)}{g^{s}}+O\left(\frac{1}{g^{s+1}}\right),
$$

where for a given $j, h_{j}(k)$ is a polynomial in $k$ of degree $2 j$. Moreover the leading coefficient of $h_{j}(k)$ is equal to $\frac{l^{j}}{2^{j} j !}$, where $l$ is the leading coefficient of the linear polynomial $p(1, j)$.

This fact is a consequence of an elementary observation. Given $m_{1}, \ldots m_{k} \in$ $\mathbb{N}$, let

$$
F_{m_{1}, \ldots, m_{k}}(d)=\sum_{\substack{x_{1}, \ldots, x_{k} \in\{1, \ldots, n\}, x_{i} \neq x_{j}}} x_{1}^{m_{1}} \cdots x_{k}^{m_{k}}
$$

is a polynomial in $d$ of degree $\left(m_{1}+1\right)+\ldots\left(m_{k}+1\right)$. Note that for $d<k$, $F_{m_{1}, \ldots, m_{k}}(d)=0$.

Now we use this observation for $f_{j}(g)=\frac{V_{g, j+1}}{4 \pi^{2}(2 g-2+j) V_{g, j}}$. In this case, (3.16) implies that $l=-1 / \pi^{2}$. Hence the result follows from (4.2) and Theorem 4.2. (ii).

\section{Asymptotics for variable $n$}

In this section we discuss the asymptotics behavior of $V_{g, n(g)}$ in case when $n(g) \rightarrow \infty$ as $g \rightarrow \infty$ and prove Theorem 1.8: if $n(g)^{2} / g \rightarrow 0$ as $g \rightarrow \infty$, then

$$
\lim _{g \rightarrow \infty} \frac{V_{g, n(g)}}{C_{g, n(g)}}=C,
$$

where $C_{g, n}=(2 g-3+n) !\left(4 \pi^{2}\right)^{2 g-3+n} g^{-1 / 2}$ and $C=\lim _{g \rightarrow \infty} \frac{V_{g, 0}}{C_{g, 0}}$.

We need the following basic lemma:

Lemma 5.1. There are universal constants $c_{0}, c_{1}, c_{2}, c_{3}, c_{4}>0$ such that for $g, n \geq 0$ the following inequalities hold:

(i) for any $k \geq 1$,

$$
c_{0} \cdot \frac{n}{2 g-2+n} \leq 1-\frac{\left[\tau_{k} \tau_{0}^{n-1}\right]_{g, n}}{V_{g, n}} \leq c_{1} \cdot \frac{n k^{2}}{2 g-2+n},
$$


(ii)

$$
\left|\frac{(2 g-2+n) V_{g, n}}{V_{g, n+1}}-\frac{1}{4 \pi^{2}}\right| \leq c_{2} \cdot \frac{n}{2 g-2+n},
$$

(iii)

$$
\frac{V_{g-1, n+4}}{V_{g, n+2}} \leq 1-c_{4} \cdot \frac{n}{2 g-2+n} .
$$

Proof. The proof follows the same lines as the proofs of formulas (3.2), (3.3) and (3.5) (see [M3] for details). First, observe that

- Recursion (III) implies

$$
\left[\tau_{k+1} \tau_{0}^{n-1}\right]_{g, n} \leq\left[\tau_{k} \tau_{0}^{n-1}\right]_{g, n} \leq V_{g, n},
$$

and we also have

$$
b \leq \frac{\left[\tau_{1} \tau_{0}^{n-1}\right]_{g, n}}{V_{g, n}}
$$

where $b=\max \left\{a_{i} / a_{i+1}\right\}, i=0,1, \ldots$;

- For $l \geq 0$

$$
\frac{l \pi^{2 l-2}}{(2 l+1) !} \geq \frac{(l+1) \pi^{2 l}}{(2 l+3) !},
$$

so that from (II) and (5.1) we have

$$
b_{0} \leq \frac{(2 g-2+n) V_{g, n}}{V_{g, n+1}} \leq b_{1},
$$

where

$$
b_{0}=b \cdot\left(\frac{1}{6}-\frac{\pi^{2}}{60}\right), \quad b_{1}=\sum_{l=1}^{\infty} \frac{l \pi^{2 l-2}}{(2 l+1) !} .
$$

- By recursion (Ia), for $n \geq 2$

$$
\frac{V_{g-1, n+4}}{V_{g, n+2}} \leq 1 .
$$

Note that in view of (5.2) and (5.3), Theorem 3.2, (ii) implies the first inequality. In order to prove $(i i)$ we will use $(i)$ and (3.15). As a result, we get

$$
\left|\frac{(2 g-2+n) V_{g, n}}{V_{g, n+1}}-\frac{1}{4 \pi^{2}}\right| \leq \sum_{l=1}^{\infty} \frac{l^{3} \pi^{2 l-2}}{(2 l+1) !} \cdot \frac{n}{2 g-2+n} .
$$

The third inequality is a simple consequence of (Ia). This bound can be obtained from the lower bound in (5.2) and (3.17).

Proof of Theorem 1.8. By Lemma 5.1,

$$
\frac{(2 g-2+n(g)) V_{g, n(g)}}{V_{g, n(g)+1}} \rightarrow \frac{1}{4 \pi^{2}}, \quad \frac{V_{g-1, n(g)+4}}{V_{g, n(g)+2}} \rightarrow 1,
$$


when $n(g) / g \rightarrow 0$ as $g \rightarrow \infty$. From definition of $C_{g, n}$ immediately follows that

$$
\frac{V_{g, n(g)}}{C_{g, n(g)}}=\frac{V_{g, n(g)}}{4 \pi^{2}(2 g-2+n(g)) V_{g, n(g)-1}} \cdot \frac{V_{g, n(g)-1}}{4 \pi^{2}(2 g-3+n(g)) V_{g, n(g)-2}} \cdots \frac{V_{g, 0}}{C_{g, 0}} .
$$

Hence, by Theorem 1.2 and Lemma 5.1. (ii) we have

$$
\begin{gathered}
\left(1-c_{2} / g\right) \cdot\left(1-2 \cdot c_{2} / g\right) \cdots\left(1-n(g) \cdot c_{2} / g\right) \cdot(C+O(1 / g)) \leq \\
\leq \frac{V_{g, n(g)}}{C_{g, n(g)}} \leq \\
\leq\left(1+c_{2} / g\right) \cdot\left(1+2 \cdot c_{2} / g\right) \cdots\left(1+n(g) \cdot c_{2} / g\right)(C+O(1 / g)),
\end{gathered}
$$

which implies the result.

Acknowledgements.

The work of MM is partially supported by an NSF grant. The work of PZ is partially supported by the RFBR grants 11-01-12092-OFI-M-2011 and 11-0100677-a, and he gratefully acknowledges the hospitality and support of MPIM (Bonn), QGM (Aarhus) and SCGP (Stony Brook). We thank Maxim Kazarian and Don Zagier for enlightening discussions.

\section{References}

[AC] E. Arbarello and M. Cornalba. Combinatorial and algebro-geometric cohomology classes on the Moduli Spaces of Curves, J. Algebraic Geometry $\mathbf{5}$ (1996), 705-709.

[DN] N. Do and P. Norbury. Weil-Petersson volumes and cone surfaces, Geom. Dedicata 141 (2009), 93-107.

[Ed] H. M. Edwards. Riemann's Zeta Function, Academic Press, 1974.

[E] B. Eynard. Recursion between Mumford volumes of moduli spaces, arXiv: 0706.4403.

[EO] B. Eynard and N. Orantin. Invariants of algebraic curves and topological expansion, Commun. Number Theory Phys. 1:2 (2007), 347-452.

[Gr] S. Grushevsky. An explicit upper bound for Weil-Petersson volumes of the moduli spaces of punctured Riemann surfaces, Mathematische Annalen. 321 (2001) 1, 1-13.

$[\mathrm{HM}]$ J. Harris and I. Morrison. Moduli of Curves. Graduate Texts in Mathematics, vol 187, Springer-Verlag, 1998.

[KMZ] R. Kaufmann, Y. Manin, and D. Zagier. Higher Weil-Petersson volumes of moduli spaces of stable n-pointed curves, Comm. Math. Phys. 181 (1996), $736-787$. 
[Ka] M. E. Kazarian. Private communication (2006).

[KL] M. E. Kazarian and S. K. Lando. An algebro-geometric proof of Witten's conjecture, J. Amer. Math. Soc. 20 (2007), 1079-1089.

[Ko] M. Kontsevich. Intersection on the moduli space of curves and the matrix Airy function, Comm. Math. Phys. 147 (1992), 1-23.

[LX1] K. Liu and H. Xu. Recursion formulae of higher Weil-Petersson volumes Int. Math. Res. Not. IMRN 5 (2009), 835-859.

[LX2] K. Liu, and H. Xu. Mirzakharni's recursion formula is equivalent to the Witten-Kontsevich theorem, Asterisque 328 (2009), 223-235.

[MZ] Yu. Manin and P. Zograf. Invertible cohomological field theories and WeilPetersson volumes, Ann. Inst. Fourier 50:2 (2000), 519-535.

[Mc] G. McShane. Simple geodesics and a series constant over Teichmüller space. Invent. Math. 132 (1998), 607-632.

[M1] M. Mirzakhani. Weil-Petersson volumes and intersection theory on the moduli space of curves, J. Amer. Math. Soc. 20:1 (2007), 1-23.

[M2] M. Mirzakhani. Simple geodesics and Weil-Petersson volumes of moduli spaces of bordered Riemann surfaces, Invent. Math. 167 (2007), 179-222.

[M3] M. Mirzakhani. Growth of Weil-Petersson volumes and random hyperbolic surfaces of large genus, arXiv:1012.2167.

[MS] Y. Mulase and P. Safnuk. Mirzakhani's recursion relations, Virasoro constraints and the KdV hierarchy, Indian Journal of Mathematics 50 (2008), 189-228.

[OP] A. Okounkov and R. Pandharipande. Gromov-Witten theory, Hurwitz numbers, and Matrix models, Proc. Symp. Pure Math. 80.1 (2009), 325-414.

[Pe] R. Penner.Weil-Petersson volumes, J. Differential Geom. 35 (1992), 559608.

[ST] G. Schumacher and S. Trapani. Estimates of Weil-Petersson volumes via effective divisors Comm. Math. Phys. 222, No.1 (2001), 1-7.

[W] E. Witten. Two-dimensional gravity and intersection theory on moduli spaces, Surveys in Differential Geometry 1 (1991), 243-269.

[Wo] S. Wolpert. On the homology of the moduli of stable curves, Ann. of Math. 118:2 (1983), 491-523.

[Z] P. Zograf. On the large genus asymptotics of Weil-Petersson volumes, arXiv:0812.0544 